\title{
RELATIVE LUBIN-TATE GROUPS
}

\author{
EHUD DE SHALIT
}

\begin{abstract}
We construct a class of formal groups that generalizes LubinTate groups. We formulate the major properties of these groups and indicate their relation to local class field theory.
\end{abstract}

The aim of this note is to introduce a certain family of formal groups generalizing Lubin-Tate groups. Although the construction, basic properties and relation with local class field theory are all similar to Lubin-Tate theory, the author is unaware of previous references to these groups. We remark, however, that they are complementary in some sense to the formal groups studied by Honda in [2]. Since we want to keep this note short, all the proofs are omitted. The reader who is acquainted with Lubin-Tate theory as in [4 or 5] will be able to supply them without any difficulties.

I would like to acknowledge my debt to K. Iwasawa. His beautiful exposition of local class field theory $[\mathbf{3}]$ motivated this note.

1. Let $k$ be a finite extension of $\mathbf{Q}_{p}, \nu: k^{\times} \rightarrow \mathbf{Z}$ the normalized valuation (normalized in the sense that $\nu\left(k^{\times}\right)=\mathbf{Z}$ ), $O$ and $\wp$ its ring of integers and maximal ideal, and $\bar{k}=0 / \wp$ the residue field, a finite field of characteristics $p$ and $q$ elements. $k^{\text {alg }}$ denotes an algebraic closure of $k$ and $k^{\text {ur }}$ the maximal unramified extension of $k$ in it. We also fix a completion of $k^{\text {alg }}, \Omega$, and let $K$ be the closure of $k^{\text {ur }}$ in it. We write $\varphi$ for the Frobenius automorphism of $k^{\mathrm{ur}} / k$, characterized by $\varphi(x) \equiv x^{q}$ $\bmod \wp^{\mathrm{ur}}$, for all $x \in \mathcal{O}^{\mathrm{ur}}$. It extends by continuity to an automorphism of $K / k$, still denoted by $\varphi$. If $k^{\prime}$ is another finite extension of $\mathbf{Q}_{p}$, the corresponding objects will be denoted by ', e.g. $\varphi^{\prime}, q^{\prime}$, etc.

If $A$ is any ring, $A\left[\left[X_{1}, \ldots, X_{n}\right]\right]$ will denote the power series ring in $X_{i}$. If $f$ and $g$ are elements of it, $f \equiv g \bmod \operatorname{deg} m$ means that the power series $f-g$ involves only monomials of degree at least $m$.

2. Fix the field $k$. For each integer $d$ let $\Sigma_{d}$ be the set of all $\xi \in k, \nu(\xi)=d$. Fix also $d>0$ and let $k^{\prime}$ be the unique unramified extension of $k$ of degree $d$. Let $\xi \in \Sigma_{d}$ and consider

$$
\mathcal{F}_{\xi}=\left\{f \in \mathcal{O}^{\prime}[[X]] \mid f \equiv \pi^{\prime} X \bmod \operatorname{deg} 2, N_{k^{\prime} / k}\left(\pi^{\prime}\right)=\xi \text { and } f \equiv X^{q} \bmod \wp^{\prime}\right\} .
$$

THEOREM 1. For each $f \in \mathcal{F}_{\xi}$ there is a unique one-dimensional commutative formal group law $F_{f} \in \mathcal{O}^{\prime}[[X, Y]]$ satisfying $F_{f}^{\varphi} \circ f=f \circ F_{f}$. In others words, $f$ is a homomorphism of $F_{f}$ to $F_{f}^{\varphi}$.

Received by the editors March 2, 1984.

1980 Mathematics Subject Classification. Primary 12B25.

Key words and phrases. Formal groups, class field theory. 
Note that if $f \in \mathcal{F}_{\xi}, f^{\varphi} \in \mathcal{F}_{\xi}$ also, and necessarily $F_{f}^{\varphi}=F_{\varphi(f)}$. If $d=1$, we are in the situation considered by Lubin and Tate. In general, we call $F_{f}$ a relative Lubin-Tate group (relative to the extension $k^{\prime} / k$ ).

3.

THEOREM 2. Let $f=\pi^{\prime} X+\cdots, g=\pi^{\prime \prime} X+\cdots$ be in $\xi_{\xi}$. Let $a \in O^{\prime}$ be an element for which $a^{\varphi-1}=\pi^{\prime \prime} / \pi^{\prime}$. Then there exists a unique power series $[a]_{f, g} \in \mathcal{O}^{\prime}[[X]]$ for which

(i) $[a]_{f, g} \equiv a X \bmod \operatorname{deg} 2$,

(ii) $[a]_{f, g}^{\varphi} \circ f=g \circ[a]_{f, g}$.

$[a]_{f, g}$ is therefore in $\operatorname{Hom}\left(F_{f}, F_{g}\right)$. If $h=\pi^{\prime \prime \prime} X+\cdots$ and $b^{\varphi-1}=\pi^{\prime \prime \prime} / \pi^{\prime \prime},[b a]_{f, h}=$ $[b]_{g, h} \circ[a]_{f, g}$. Moreover, the map $a \mapsto[a]_{f, g}$ is an additive injective homomorphism from $\left\{a \in \mathcal{O}^{\prime} \mid a^{\varphi-1}=\pi^{\prime \prime} / \pi^{\prime}\right\}$ to $\operatorname{Hom}\left(F_{f}, F_{g}\right)$. If $f=g$ it is a ring homomorphism $0 \rightarrow \operatorname{End}\left(F_{f}\right), a \mapsto[a]_{f}=[a]_{f, f}$.

COROLlaRY. If $f, g \in \mathcal{F}_{\xi}, F_{f}$ and $F_{g}$ are isomorphic.

4. Pick $\xi, \xi^{\prime} \in \Sigma_{d}$ and set $v=\xi / \xi^{\prime}$. Let $u$ be a unit of $k^{\prime}$ such that $N_{k^{\prime} / k}(u)=v$, $\theta_{1} \in K$ such that $\theta_{1}^{\varphi} / \theta_{1}=u$, and $f \in \mathcal{F}_{\xi}$.

THEOREM 3. There exists a unique power series $\theta(X) \in O_{K}[[X]]$ satisfying

(i) $\varphi^{d}(\theta)=\theta \circ[v]_{f}$,

(ii) $\theta(X) \equiv \theta_{1} X \bmod \operatorname{deg} 2$.

Put $f^{\prime}=\theta^{\varphi} \circ f \circ \theta^{-1}$. Then $f^{\prime} \in \mathcal{F}_{\xi^{\prime}}$ and $\theta$ is an isomorphism of $F_{f}$ onto $F_{f^{\prime}}$ over $O_{K}$.

5.

Definition. For $i \geq 0$ and $f \in \mathcal{F}_{\xi}$, let $f^{(i)}=\varphi^{i-1}(f) \circ \cdots \circ \varphi(f) \circ f$. Then $f^{(i)} \in \operatorname{Hom}\left(F_{f}, F_{f}^{\varphi^{i}}\right)$ and (if $\left.\xi \in \Sigma_{d}\right) f^{(d)}=[\xi]_{f} \in \operatorname{End}\left(F_{f}\right)$. Note also that $\varphi^{j}\left(f^{(i)}\right) \circ f^{(j)}=f^{(i+j)}$.

Let $M$ be the valuation ideal of $\Omega$, and $M_{f}$ the commutative group whose underlying set is $M$ and the addition is given by $F_{f}$. With $\xi \in \Sigma_{d}, f \in \mathcal{F}_{\xi}$ and $\pi$ a prime element of 0 , define for any $n \geq 0$

$$
\begin{aligned}
W_{f}^{n} & =\left\{\alpha \in M_{f} \|[a]_{f}(\alpha)=0 \text { for all } a \in \wp^{n+1}\right\} \\
& =\left\{\alpha \in M_{f} \|\left[\pi^{n+1}\right](\alpha)=0\right\} \\
& =\operatorname{Ker}\left(f^{(n+1)}: M_{f} \rightarrow M_{\varphi^{n+1}(f)}\right) .
\end{aligned}
$$

PROPOSITION 1. (i) $W_{f}^{n}$ is a finite sub-O-module of $M_{f}$ and has $q^{n+1}$ elements. $W_{f}^{n} \subseteq W_{f}^{n+1}$.

(ii) If $\alpha \in W_{f}^{n}$ but $\alpha \notin W_{f}^{n-1}, a \mapsto[a]_{f}(\alpha)$ gives an isomorphism $0 / \wp^{n+1} \cong W_{f}^{n}$.

(iii) $W_{f}=\bigcup W_{f}^{n} \cong k / O$ (noncanonically) and is the set of all $O$-torsion in $M_{f}$.

6. Coleman's norm operator (see [1]). Let $R=O^{\prime}[[X]], \xi \in \Sigma_{d}$, and $f \in \xi_{\xi}$.

PROPOSITION 2. There exists a unique multiplicative operator $\mathcal{N}: R \rightarrow R(\mathcal{N}=$ $\mathcal{N}_{f}$, to emphasize the dependence on $f$ ), such that

$$
(\mathcal{N} h) \circ f(X)=\prod_{\alpha \in W_{f}^{0}} h\left(X[+]_{f} \alpha\right) \quad \forall h \in R .
$$


It enjoys the additional properties:

(i) $\mathcal{N} h \equiv h^{\varphi} \bmod \wp^{\prime}$,

(ii) $\mathcal{N}_{f} \varphi=\varphi \circ \mathcal{N}_{f} \circ \varphi^{-1}$, i.e. $\mathcal{N}_{f} \varphi\left(h^{\varphi}\right)=\left(\mathcal{N}_{f} h\right)^{\varphi}$,

Then

(iii) Let $\mathcal{N}_{f}^{(i)} h=\mathcal{N}_{\varphi^{i-1}(f)} \circ \cdots \circ \mathcal{N}_{\varphi(f)} \circ \mathcal{N}_{f}(h)$.

$$
\left(\mathcal{N}_{f}^{(i)} h\right) \circ f^{(i)}(X)=\prod_{\alpha \in W_{f}^{i-1}} h\left(X[+]_{f} \alpha\right) .
$$

(iv) If $h \in R$ and $h \equiv 1 \bmod \wp^{i}(i \geq 1)$, then $\mathcal{N} h \equiv 1 \bmod \wp^{i+1}$.

7.

Proposition 3. The field $k^{\prime}\left(W_{f}^{n}\right)$ is the same for all $f \in \mathcal{F}_{\xi}$. Call it $k_{\xi}^{n}$, and put $k_{\xi}^{-1}=k^{\prime}$. Then for $n \geq 0, k_{\xi}^{n}$ is a totally ramified extension of $k^{\prime}$ of degree $(q-1) q^{n}$, and it is abelian over $k$. Any $\alpha$ in $W_{f}^{n}$ but not in $W_{f}^{n-1}$, for any $f \in \mathcal{F}_{\xi}$, generates $k_{\xi}^{n}$ over $k^{\prime}$ and is a prime element for it.

Much more can be said about those fields (see $\S 10$ ).

\section{Coleman power series $[1]$.}

THEOREM 4. Fix $\xi \in \Sigma_{d}, f \in \mathcal{F}_{\xi}$ and $\alpha \in W_{\varphi^{-n}(f)}^{n}, \alpha \notin W_{\varphi^{-n}(f)}^{n-1}$. For $0 \leq i \leq n$ let $\alpha_{i}=\left(\varphi^{-n}(f)\right)^{(n-i)}(\alpha)=\varphi^{-i-1}(f) \circ \cdots \circ \varphi^{-n}(f)(\alpha) \in W_{\varphi^{-i}(f)}^{i}$. Let $c$ be a unit of $k_{\xi}^{n}$ and $c_{i}=N_{n, i}(c)\left(N_{n, i}\right.$ denoting the norm from $k_{\xi}^{n}$ to $\left.k_{\xi}^{i}\right)$. Then there is a power series $g$ in $R$ such that

$$
\varphi^{-i}(g)\left(\alpha_{i}\right)=c_{i} \quad(0 \leq i \leq n) .
$$

COROLlaRY. Suppose $\alpha_{i}$ is an element of $W_{\varphi^{-i}(f)}^{i}$ not in $W_{\varphi^{-i}(f)}^{i-1}(i \geq 0)$ and $f^{\varphi^{-i}}\left(\alpha_{i}\right)=\alpha_{i-1}$. Suppose also $c_{0}, c_{1}, \ldots$ is a norm-compatible sequence of units in $k_{\xi}^{i}$, i.e. $N_{n, i}\left(c_{n}\right)=c_{i}$. Then there exists a unique $g$ in $R$ such that $g^{\varphi^{-i}}\left(\alpha_{i}\right)=c_{i}$ for all $i$.

9.

EXAMPLE. Let $K$ be a quadratic imaginary field, let $F$ be a finite extension of $K$, and let $E$ be an elliptic curve defined over $F$ with complex multiplication by the full ring of integers of $K$. As explained in [6], if we choose a Weierstrass model of $E$ over the integers of $F$ we get a formal group law $\hat{E}(X, Y)$ defined over the ring generated (over $\mathbf{Z}$ ) by the coefficients in the Weierstrass equation. Let $p$ be a prime of $K$ and $P$ a prime of $F$ dividing $p$. Assume $E$ has good reduction at $P$, and that $P$ is not ramified in $F / K$. It is then a consequence of the theory of complex multiplication that $\hat{E}$, as a formal group defined over $O_{P}$ (the integers of $F_{P}$ ), is a relative Lubin-Tate group with respect to the (unramified) extension $F_{P} / K_{p}$.

10. The relation between Lubin-Tate groups and local class field theory can now be easily generalized. A full description of it (and actually derivation of local class field theory from the formal group point of view) can be found in [3]. We only make the following remarks. The fields $k_{\xi}=\bigcup k_{\xi}^{n}=k^{\prime}\left(W_{f}\right)$ (for any $f \in \mathcal{F}_{\xi}$ ) are the maximal abelian extensions of $k$ with residue field equal to the extension of degree $d$ of $\bar{k}$. They are distinct for different $\xi$ as can be seen from the observation that the group of universal norms from $k_{\xi}$ to $k$ is just the cyclic group generated by $\xi$. 
If $\xi \in \Sigma_{1}^{d}$, i.e. is a $d$ th power in $k$, then $\mathcal{F}_{\xi}$ contains an $f$ from $O[[X]]$. In this case $k_{\xi}$ is the compositum of a totally ramified extension of $k$ and $k^{\prime}$. However, this is not always the case, because $\Sigma_{d} \neq \Sigma_{1}^{d}$ in general.

\section{REFERENCES}

1. R. Coleman, Division values in local fields, Invent. Math. 53 (1979), 91-116.

2. T. Honda, Formal groups and zeta functions, Osaka. J. Math. 5 (1968) 199-213.

3. K. Iwasawa, Local class field theory, Oxford Univ. Press, London (to appear).

4. J. Lubin and J. Tate, Formal complex multiplication in local fields, Ann. of Math. (2) 81 (1965), 380-387.

5. J. P. Serre, Local class field theory, Algebraic Number Theory (Cassels and Frohlich, eds.), Academic Press, New York, 1967.

6. J. Tate, The arithmetic of elliptic curves, Invent. Math 23 (1974), 179-206.

Department of Mathematics, Fine hall, Princeton University, Princeton, NEW JERSEY 08540

Current address: Department of Mathematics, Science Center, 1 Oxford Street, Harvard University, Cambridge, Massachusetts 02138 